\title{
The m6A demethylase FTO promotes esophageal cancer progression through YTHDF1-dependent posttranscriptional silencing of AKT3
}

\section{Chunbao Zang}

The First Affiliated Hospital of USTC: Anhui Provincial Hospital

\section{Fangfang Zhao}

The First Affiliated Hospital of USTC: Anhui Provincial Hospital

Dabing Huang

The First Affiliated Hospital of USTC: Anhui Provincial Hospital

\section{Lingsuo Kong}

The First Affiliated Hospital of USTC: Anhui Provincial Hospital

\section{Minghua Xie}

The First Affiliated Hospital of USTC: Anhui Provincial Hospital

\section{Yiao Tan}

The First Affiliated Hospital of USTC: Anhui Provincial Hospital

\section{Youguang Pu ( $\nabla$ puyouguang@126.com )}

Anhui Provincial Hospital https://orcid.org/0000-0002-6056-4831

\section{Research Article}

\section{Keywords:}

Posted Date: July 27th, 2021

DOI: https://doi.org/10.21203/rs.3.rs-589523/v2

License: (c) (i) This work is licensed under a Creative Commons Attribution 4.0 International License. Read Full License 


\section{Abstract}

Background : $\mathrm{N} 6$-methyladenosine ( $\mathrm{m} 6 \mathrm{~A}$ ) is the most abundant modification in eukaryotic messenger RNAs (mRNAs), and plays important roles in many bioprocesses. However, its functions in esophageal cancer remain elusive. Methods : Methylated RNA immunoprecipitation sequencing (MeRIP-seq) and transcriptomic RNA sequencing (RNA-seq) were used to screen the target genes of FTO. Western blot, quantitative real-time PCR (RT-qPCR) and immunohistochemical (IHC) were used to detect

FTO expression in cell lines and patient tissues. The biological functions of FTO were investigated in vitro and in vivo. RNA pull-down and RNA immunoprecipitation assays were conducted to explore the specific binding of target genes. Results : We discovered that the RNA demethylase FTO was significantly upregulated in esophageal cancer patients. Knockdown of FTO drastically reduced esophageal cancer cells (ESCCs) proliferation, migration, invasion, and apoptosis. On the other hand, overexpression of FTO significantly promoted ESCCs growth and invasion. Moreover, we found that the $\mathrm{m} 6 \mathrm{~A}$ methyltransferase METTL14 negatively correlates with FTO function on esophageal cancer progression. By using transcriptome-wide $\mathrm{m} 6$ A-Seq and RNA-Seq assays, we identified AKT3 is the target of FTO, which acts in concert in esophageal cancer tumorigenesis and metastasis. Moreover, loss and gain functional studies confirm that YTHDF1 mediates m 6 A-increased translation of AKT3 mRNA. Conclusion : Our results uncovered an METTL14/FTO/YTHDF1/AKT3 signaling network that regulates the esophageal cancer progression. 